\title{
Thyroid hormone treatment among pregnant women with subclinical hypothyroidism: US national assessment
}

\author{
(c) (1) (8) OPEN ACCESS
}

\begin{abstract}
Spyridoula Maraka assistant professor of medicine ${ }^{12}$, Raphael Mwangi statistical programmer analyst ${ }^{3}$, Rozalina G McCoy assistant professor of medicine ${ }^{34}$, Xiaoxi Yao research associate ${ }^{3}$, Lindsey R Sangaralingham assistant professor of health services research ${ }^{3}{ }^{6}$, Naykky M Singh Ospina assistant professor of medicine ${ }^{27}$, Derek T O'Keeffe assistant professor of medicine ${ }^{89}$, Ana E Espinosa De Ycaza instructor in medicine ${ }^{9}$, Rene Rodriguez-Gutierrez postdoctoral researcher ${ }^{2}$ ${ }^{10}$, Charles C Coddington III professor of medicine ${ }^{11}$, Marius N Stan assistant professor of medicine ${ }^{9}$, Juan P Brito assistant professor of medicine ${ }^{29}$, Victor M Montori professor of medicine ${ }^{29}$
\end{abstract}

\begin{abstract}
'Division of Endocrinology and Metabolism, Center for Osteoporosis and Metabolic Bone Diseases, University of Arkansas for Medical Sciences and the Central Arkansas Veterans Health Care System, Little Rock, AR 72205, USA; ${ }^{2}$ Knowledge and Evaluation Research Unit in Endocrinology (KER_Endo), Mayo Clinic, Rochester, MN 55905, USA; ${ }^{3}$ Robert D. and Patricia E. Kern Center for the Science of Health Care Delivery, Mayo Clinic, Rochester, MN 55905, USA; ${ }^{4}$ Division of Primary Care Internal Medicine, Department of Medicine, Mayo Clinic, Rochester, MN 55905, USA; ${ }^{5}$ Division of Health Care Policy and Research, Department of Health Services Research, Mayo Clinic, Rochester, MN 55905, USA; ${ }^{6}$ Optum Labs, Cambridge, MA 02142, USA; ${ }^{7}$ Division of Endocrinology, Department of Medicine, University of Florida, Gainesville, FL 32610, USA; ${ }^{8}$ Division of Endocrinology, Department of Medicine, National University of Ireland, Galway, Ireland; ' ${ }^{\circ}$ Division of Endocrinology, Diabetes, Metabolism, and Nutrition, Mayo Clinic, Rochester, MN 55905, USA; ${ }^{10}$ Division of Endocrinology, Department of Internal Medicine, University Hospital "Dr. Jose E. Gonzalez," Autonomous University of Nuevo Leon, Monterrey, 64460, Mexico; ${ }^{11}$ Department of Obstetrics and Gynecology, Mayo Clinic, Rochester, MN 55905 ,
\end{abstract} USA

\begin{abstract}
Objective To estimate the effectiveness and safety of thyroid hormone treatment among pregnant women with subclinical hypothyroidism.

Design Retrospective cohort study.

Setting Large US administrative database between 1 January 2010 and 31 December 2014.

Participants 5405 pregnant women with subclinical hypothyroidism, defined as untreated thyroid stimulating hormone (TSH) concentration 2.5-10 mIU/L

Exposure Thyroid hormone therapy.

Main outcome measure Pregnancy loss and other pre-specified maternal and fetal pregnancy related adverse outcomes.
\end{abstract}

Results Among 5405 pregnant women with subclinical hypothyroidism, 843 with a mean pre-treatment TSH concentration of 4.8 (SD 1.7) $\mathrm{mIU} / \mathrm{L}$ were treated with thyroid hormone and 4562 with a mean baseline TSH concentration of $3.3(\mathrm{SD} 0.9) \mathrm{mIU} / \mathrm{L}$ were not treated $(\mathrm{P}<0.01)$. Pregnancy loss was significantly less common among treated women $(n=89 ; 10.6 \%)$ than among untreated women $(\mathrm{n}=614 ; 13.5 \%)(\mathrm{P}<0.01)$. Compared with the untreated group, treated women had lower adjusted odds of pregnancy loss (odds ratio $0.62,95 \%$ confidence interval 0.48 to 0.82 ) but higher odds of preterm delivery $(1.60,1.14$ to 2.24$)$, gestational diabetes (1.37, 1.05 to 1.79$)$, and pre-eclampsia (1.61, 1.10 to 2.37); other pregnancy related adverse outcomes were similar between the two groups. The adjusted odds of pregnancy loss were lower in treated women than in untreated women if their pre-treatment TSH concentration was $4.1-10 \mathrm{mIU} / \mathrm{L}$ (odds ratio $0.45,0.30$ to 0.65 ) but not if it was $2.5-4.0$ $\mathrm{mIU} / \mathrm{L}(0.91,0.65$ to 1.23$)(\mathrm{P}<0.01)$.

Conclusion Thyroid hormone treatment was associated with decreased risk of pregnancy loss among women with subclinical hypothyroidism, especially those with pre-treatment TSH concentrations of 4.1-10 mIU/L. However, the increased risk of other pregnancy related adverse outcomes calls for additional studies evaluating the safety of thyroid hormone treatment in this patient population. 


\section{Introduction}

Changes in thyroid physiology during pregnancy result in the normal range of thyroid stimulating hormone (TSH) being lower than among non-pregnant adults. International guidelines advocate using population based reference ranges; however, if these are unavailable the recommended fixed upper threshold for TSH concentration is $2.5 \mathrm{mIU} / \mathrm{L}$ during the first trimester and $3.0 \mathrm{mIU} / \mathrm{L}$ during the second and third trimesters. ${ }^{1}$

According to these diagnostic criteria, subclinical

hypothyroidism, defined as an elevated TSH concentration with concurrent normal thyroid hormone concentrations, is estimated to affect up to $15 \%$ of pregnancies in the US and $14 \%$ in Europe. ${ }^{23}$ This represents a fivefold increase in prevalence compared with the $2-3 \%$ prevalence of subclinical hypothyroidism before these criteria were established, raising the possibility of overdiagnosis of subclinical hypothyroidism and discussions at the 2016 Endocrine Society meeting about increasing the TSH cut-off limit to $4.0 \mathrm{mIU} / \mathrm{L}$ in the upcoming American Thyroid Association guidelines.

Observational studies have examined the effect of subclinical hypothyroidism on pregnancy related outcomes, and it has been associated with multiple adverse outcomes in many, but not all, studies. ${ }^{4-6}$ A recent meta-analysis of 18 cohort studies found that pregnant women with untreated subclinical hypothyroidism are at higher risk for pregnancy loss, placental abruption, premature rupture of membranes, and neonatal death compared with euthyroid women. ${ }^{7}$ Current guidelines recommend levothyroxine treatment in pregnant women with subclinical hypothyroidism. ${ }^{89}$ However, insufficient evidence exists to show that thyroid hormone treatment improves clinical outcomes in pregnant women with subclinical hypothyroidism. Moreover, in theory, full implementation of these guidelines would lead to levothyroxine treatment being started in up to 600000 pregnant women in the US each year, ${ }^{10}$ increasing the cost of healthcare and the burden on patients. The degree to which these recommendations have been implemented in the US is unknown. We used data from a large national US dataset to determine the prevalence, effectiveness, and safety of thyroid hormone treatment among pregnant women with subclinical hypothyroidism.

\section{Methods \\ Dataset}

We did a retrospective analysis of data from the OptumLabs Data Warehouse, an administrative claims database that includes privately insured and Medicare Advantage enrollees throughout the US. ${ }^{11}$ The database contains health information on more than 100 million people, spanning more than 20 years, and includes people from geographically diverse regions across the US and of all races/ethnicities and ages and both sexes. ${ }^{12}$ The included data comprise covered professional (eg, physician), facility (eg, hospital), and outpatient prescription drug services. Medical claims include ICD-9-CM (international classification of diseases, 9th revision, clinical modification) diagnosis codes and procedure codes; Current Procedural Terminology, version 4 (CPT-4) procedure codes; Healthcare Common Procedure Coding System procedure codes; site of service codes; and provider specialty codes. Laboratory data, available for a subset of people on the basis of data sharing agreements, include test names, logical observation identifiers names and codes, and test results. We used methods compliant with the Health Insurance Portability and Accountability Act of 1996 Study to access data.

\section{Study population}

Using available laboratory data, we identified all women aged 18-55 years who had subclinical hypothyroidism defined as TSH concentration 2.5-10 mIU/L within four weeks before or three months after a first medical claim indicating a pregnancy visit (supplementary table A) between 1 January 2010 and 31 December 2014 (fig $1 \Downarrow$ ). We excluded women who did not have uninterrupted medical and pharmacy coverage for a 12 month period before (to ascertain comorbidities and previous thyroid hormone use) and at least 42 weeks after (to ascertain pregnancy outcomes) the date of TSH measurement. If women had thyroxine concentrations checked within a week of the TSH test and found to be low (free thyroxine $<0.8 \mathrm{ng} / \mathrm{dL}$ and/or total thyroxine $<7.5 \mu \mathrm{g} / \mathrm{dL}$ ), we excluded them from analysis because this indicates overt hypothyroidism rather than subclinical hypothyroidism. We also excluded women with multiple gestation pregnancies (ascertained by ICD-9-CM codes; supplementary table B), because higher human chorionic gonadotropin concentrations in multiple gestation pregnancies lower the TSH reference range compared with singleton pregnancies and can therefore confound analyses. ${ }^{13}$ We excluded women who used drugs affecting thyroid function (amiodarone, methimazole, and propylthiouracil), were treated with thyroid hormone during the 12 month baseline period, had no codes indicating end of pregnancy within 42 weeks of the index TSH date, or had a code indicating end of pregnancy (supplementary table C) before the index TSH date (TSH test performed after the end of pregnancy).

We recorded patients' baseline characteristics at the time of the index TSH test, including age, race/ethnicity, household income, and census region. We classified ethnicity as non-Hispanic white (white), non-Hispanic black (black), Asian, or Hispanic. ${ }^{14}$ To quantify the baseline comorbidity burden, we used the Charlson comorbidity index for one calendar year before the index test date, using ICD-9-CM diagnoses included in administrative claims. The Charlson index is a widely used measure that weights comorbid conditions by the strength of their association with one year mortality. ${ }^{15} \mathrm{We}$ also recorded information on personal history of thyroid disease (not treated with thyroid hormone), obesity, diabetes mellitus, hypertension, and recurrent pregnancy loss based on ICD-9-CM codes (supplementary table D).

\section{Identification of exposure}

We divided the cohort into two groups depending on whether they were treated with thyroid hormone after the index TSH date. Treatment status was ascertained by pharmacy claims. Thyroid hormone treatments included levothyroxine, liothyronine, and thyroid extract formulations.

\section{Identification of adverse outcomes}

We identified adverse outcomes of pregnancy by using ICD-9-CM codes during evaluation and management face-to-face clinical encounters, as detailed in supplementary table E. The primary outcome was pregnancy loss (miscarriage and stillbirth). Secondary outcomes included preterm delivery, preterm labor, premature rupture of membranes, placental abruption, gestational diabetes, gestational hypertension, pre-eclampsia, poor fetal growth, and tachycardia.

\section{Patient involvement}

No patients were involved in setting the research question or the outcome measures, nor were they involved in developing plans for design or implementation of the study. No patients 
were asked to advise on interpretation or writing up of results. There are no plans to disseminate the results of the research to study participants or the relevant patient community.

\section{Statistical analysis}

We present summary data of patients' baseline characteristics as frequency (percentage) for categorical variables and mean (standard deviation) or median (interquartile range) for continuous variables. We assessed differences between categorical variables by using $\chi^{2}$ tests and between continuous variables by using $t$ tests. Multivariable logistic regression assessed the association of thyroid hormone treatment with adverse pregnancy outcomes while controlling for potential confounders of TSH concentration, age, ethnicity, income, Charlson index, obesity, history of thyroid disease, and hypertension. When examining outcomes of gestational hypertension and pre-eclampsia, we did not include history of hypertension in the regression model as these diagnoses are mutually exclusive. When examining the outcome of pregnancy loss, we also controlled for history of recurrent pregnancy loss, which is known to be a strong risk factor for this outcome. ${ }^{16} \mathrm{We}$ did a predetermined sensitivity analysis of treatment effect on all pregnancy related adverse outcomes stratified by a pre-treatment TSH concentration of $4.0 \mathrm{mIU} / \mathrm{L}$, because this TSH concentration will be used as a treatment threshold in the upcoming American Thyroid Association guidelines for subclinical hypothyroidism during pregnancy. Results are reported as odds ratios and 95\% confidence intervals, with untreated women as the reference group. We considered a $\mathrm{P}$ value below 0.05 to be statistically significant, and all testing was two sided. We used SAS software version 9.4 for all statistical analyses.

\section{Results}

\section{Baseline characteristics}

We identified 5405 women with subclinical hypothyroidism between 1 January 2010 and 31 December 2014. Of these, 843 (15.6\%) women started thyroid hormone treatment; 832 (98.7\%) with levothyroxine at a median dose of $50 \mu \mathrm{g}$ (interquartile range 25-62.5 $\mu \mathrm{g}$; mean 52.8 (SD 31.8) $\mu \mathrm{g}$; range 25-300 $\mu \mathrm{g}$ ), $7(0.8 \%)$ with thyroid extract formulation, and $4(0.5 \%)$ with a combination of levothyroxine and liothyronine. The remaining 4562 (84.4\%) women were not treated with thyroid hormone. The percentage of women treated increased each year of the study: $12 \%(136 / 1100)$ of pregnant women with subclinical hypothyroidism were treated in 2010, 15\% (161/1094) in 2011, $15 \%(160 / 1040)$ in $2012,17 \%(214 / 1251)$ in 2013 , and $19 \%$ $(172 / 920)$ in 2014. There was also geographic variation in the prescription of thyroid hormone, with significantly more women receiving treatment in the Northeast and West than in the Midwest and South regions of the US $(\mathrm{P}<0.01)$. The median time between TSH test and starting thyroid hormone treatment was 11 (interquartile range 4-15) days, and the median time between TSH test and childbirth was 30.3 (25.4-32.7) weeks. Adherence to thyroid hormone treatment was good, with a mean proportion of $88 \%$ of days covered. Of the 843 women who were treated, $719(85.3 \%)$ had at least one subsequent TSH test result during the follow-up period. Using the last TSH measurement, $130(18.0 \%)$ women had a TSH concentration above $3 \mathrm{mIU} / \mathrm{L}$.

Table $1 \Downarrow$ shows patients' baseline characteristics. The groups were similar with regard to mean age, but the untreated group had a higher proportion of patients in the 18-24 year age group than did the treated group. The groups were not different with regard to Charlson index, obesity, or prevalence of pre-pregnancy diabetes. Treated patients had higher pre-treatment mean TSH concentration (4.8 (SD 1.7) mIU/L) and more prevalent pre-existing thyroid disease (eg, thyroid nodules: $\mathrm{n}=52 ; 6.2 \%)$ compared with untreated patients $(3.3$ (SD 0.9) $\mathrm{mIU} / \mathrm{L}$ and $\mathrm{n}=153 ; 3.4 \%$, respectively) $(\mathrm{P}<0.01)$. Figure $2 \Downarrow$ shows the distribution of TSH in the two groups. Treated patients had a greater prevalence of past recurrent pregnancy loss $(n=23 ; 2.7 \%)$ and hypertension $(n=22 ; 2.6 \%)$ compared with untreated patients $(n=49 ; 1.1 \%$ and $n=221 ; 4.8 \%$, respectively) $(\mathrm{P}<0.01)$.

\section{Pregnancy related adverse outcomes}

Pregnancy loss was significantly less common among treated women $(n=89 ; 10.6 \%)$ than among untreated women $(n=614$; $13.5 \%)(\mathrm{P}<0.01)$, with a median time from TSH measurement to pregnancy loss of 3.3 (IQR 1.6-5.6) weeks. After adjustment for potential confounders of pregnancy loss-specifically age, TSH concentration, ethnicity, income, Charlson index, hypertension, obesity, history of thyroid disease, and history of pregnancy loss - treated women had a $38 \%$ lower odds of pregnancy loss compared with untreated women (odds ratio $0.62,95 \%$ confidence interval 0.48 to 0.82 ).

Other pregnancy related adverse outcomes were also differentially affected by treatment status, as shown in table $2 \Downarrow$. Compared with untreated women, treated women had higher adjusted odds of preterm delivery (odds ratio 1.60, 1.14 to 2.24), gestational diabetes $(1.37,1.05$ to 1.79$)$, and pre-eclampsia (1.61, 1.10 to 2.37$)$. The higher odds of preterm delivery in treated women persisted even after adjustment for gestational diabetes, gestational hypertension, and pre-eclampsia (odds ratio $1.45,1.03$ to 2.05 ). Other pregnancy related adverse outcomes were similar in the two groups. Of the 654 (77.6\%) treated women who had a repeat TSH test during pregnancy, $18(2.8 \%)$ had a serum TSH concentration below $0.1 \mathrm{mIU} / \mathrm{L}$ but none had tachycardia. We found no difference in the frequency of tachycardia between groups (treated $2.1 \%$ versus untreated $2 \% ; \mathrm{P}=0.05$ ).

We found a significant interaction between the pre-treatment TSH concentration (continuous variable) and effect of treatment on pregnancy loss (table $3 \Downarrow$ ). We did a subgroup analysis of treatment effect on all pregnancy related adverse outcomes stratified by pre-treatment TSH concentration (table $4 \Downarrow$ ). The odds of pregnancy loss were significantly lower among treated women with pre-treatment TSH 4.1-10 mIU/L (odds ratio 0.45, 0.30 to 0.65 ) but not those with pre-treatment TSH 2.5-4.0 $\mathrm{mIU} / \mathrm{L}$ compared with untreated women $(0.91,0.65$ to 1.23$)$ $(\mathrm{P}<0.01$ for interaction). Conversely, the risk of gestational hypertension was significantly higher among treated than untreated women with pre-treatment TSH 2.5-4.0 mIU/L (odds ratio $1.76,1.13$ to 2.74 ) but was comparable between treated and untreated women with pre-treatment TSH 4.1-10.0 mIU/L $(\mathrm{P}=0.04$ for interaction). We found no significant interaction between pre-treatment TSH concentration and effect of thyroid hormone use on any other adverse outcome.

A sensitivity analysis restricted the treated study cohort to women treated with levothyroxine only. None of the results were affected (supplementary tables F and G), with the exception of the interaction between pre-treatment TSH concentration and the effect of thyroid hormone use on the risk of gestational hypertension ( $\mathrm{P}=0.05$ for interaction).

Nearly $80 \%(n=673)$ of women in the treated cohort were adherent to prescribed thyroid hormone treatment, as defined by a proportion of days covered of at least $80 \%$, whereas $20.2 \%$ 
$(\mathrm{n}=170)$ had lower levels of adherence. The adherent group was older (mean age of 32 (SD 4.4) years) than the non-adherent group (mean age 30.6 ( $\mathrm{SD} 5.4)$ years) $(\mathrm{P}<0.01)$. The two groups also had different household income distribution $(\mathrm{P}=0.03)$, but they were not different with regards to other baseline characteristics (supplementary table $\mathrm{H}$ ). An additional sensitivity analysis for pregnancy related adverse outcomes affected by treatment restricted the treated study cohort to the patients who were adherent. There was no difference compared with the main analysis results presented in tables 2 and $4 \Downarrow$, except for a change in significance of some clinical outcomes that could be due to the smaller sample size (supplementary table I and supplementary table J, respectively).

\section{Discussion}

Only $16 \%$ of pregnant women with subclinical hypothyroidism included in this large national US cohort received thyroid hormone treatment. Clinical practice guidelines on treatment of subclinical hypothyroidism during pregnancy were based on insufficient evidence, and our findings show the effect of using thyroid hormone replacement on pregnancy loss and other pregnancy related adverse events. We found that use of thyroid hormone was associated with decreased risk of pregnancy loss, but it was also associated with increased risk of preterm delivery, gestational diabetes, and pre-eclampsia. Thyroid hormone treatment had no apparent effect on other important adverse outcomes. Moreover, the benefit of thyroid hormone use on pregnancy loss was observed only among women with pre-treatment TSH concentrations of 4.1-10.0 mIU/L, not those with concentrations of 2.5-4.0 mIU/L, raising questions about the current guideline recommended threshold of $2.5 \mathrm{mIU} / \mathrm{L}$ for treating subclinical hypothyroidism when population reference ranges are unavailable.

\section{Comparison with other studies}

Our study adds to the existing literature assessing the risks and benefits of levothyroxine treatment in patients with subclinical hypothyroidism, which is hindered by imprecision, moderate risk of bias, and lack of generalizability to patients outside of tertiary care referral centers. Although no published randomized clinical trials have evaluated the effect of thyroid hormone use on obstetric outcomes in subclinical hypothyroidism during pregnancy, small observational studies have suggested a potential benefit. A prospective study in China screened women in the first trimester of pregnancy for thyroid dysfunction and found an association between subclinical hypothyroidism and pregnancy loss, but it did not show any benefits of levothyroxine treatment. ${ }^{17}$ Another prospective study in Italy that included pregnant women with serum TSH above $2.5 \mathrm{mIU} / \mathrm{L}$ and positive thyroid peroxidase antibody concentrations found that the proportion of women with at least one adverse obstetric or neonatal outcome was significantly higher in the untreated subgroup than in a subgroup of women at similar risk who were treated. This suggested a possible benefit from levothyroxine treatment. ${ }^{18}$ These studies were limited by a small sample size and number of events leading to imprecision and failed to adjust for covariates of pregnancy complications. Our group recently published a single center study performed at an academic tertiary care center and including predominantly white pregnant women with subclinical hypothyroidism. We found that levothyroxine treatment in this setting was associated with a decreased risk of low birth weight and low Apgar score. Although the available data on multiple potential confounders, most notably socioeconomic measures and obstetric comorbid conditions, allowed us to adjust the analyses appropriately, this study was also limited by its retrospective, observational design as well as the small size. ${ }^{19}$

Thus, although clinical guidelines recommend thyroid hormone treatment for pregnant women with subclinical hypothyroidism, these recommendations have been supported by weak and inconsistent scientific evidence, potentially limiting their implementation in routine clinical practice. Despite the recommendations of the American Thyroid Association in 2011 and the Endocrine Society in 2012 for treatment of subclinical hypothyroidism during pregnancy, ${ }^{18}$ less than $16 \%$ of our cohort received thyroid hormone by 2014 . Moreover, $1.2 \%$ of the treated women received thyroid hormone preparations other than levothyroxine, against current recommendations. ${ }^{18}$ The cause of this discrepancy is likely multifactorial, including a lack of familiarity with the guidelines among clinicians, ${ }^{20}$ paucity of trustworthy evidence to support such recommendations, ${ }^{21}$ inconsistencies in practice among endocrinologists, family medicine physicians, and obstetricians, ${ }^{22}$ and non-adherence to levothyroxine by patients. ${ }^{23}$

The association of thyroid hormone use with clinically decreased risk of pregnancy loss in our study is consistent with previous reports. ${ }^{17-19}$ From a biologic standpoint, studies on human placental tissue suggest that the optimal concentration of thyroid hormone is needed for it to exert its maximal stimulatory action on trophoblast endocrine function. ${ }^{24}$ Therefore, pregnancy loss in early pregnancy during the state of hypothyroidism may represent a direct consequence of inadequate thyroid hormone availability at the level of placental trophoblasts, followed by diminished expression of trophoblast endocrine function. This evidence lends biologic plausibility to the observations described here. However, we also detected an association of thyroid hormone treatment with increased risk of adverse pregnancy outcomes (preterm delivery, gestational diabetes, and pre-eclampsia). Although the increase in absolute risk was small, this finding is not congruent with an overall benefit of thyroid hormone treatment and raises concerns about overtreatment of pregnant women with TSH concentrations of 2.5-4.0 mIU/L. These results might reflect treatment decisions based on the physician's perception of risk, whereby women with a higher risk of pregnancy complications were more likely to be offered thyroid hormone. This bias, however, would strengthen the findings of benefit. Finally, if thyroid hormone treated women experiencing pregnancy complications sought medical attention more often than untreated women did, then adverse pregnancy outcomes would be more likely to be diagnosed among these women, which would bias the results toward an increased risk with thyroid hormone exposure. Alternatively, treated women might have been more adherent to follow-up and treatment recommendations resulting in, or associated with, lower rates of adverse outcomes compared with non-treated women, who might have presented later to care; these and other changes in behavior due to treatment status could affect our adverse outcome rates.

\section{Policy implications}

Both clinicians and patients with subclinical hypothyroidism in pregnancy still face uncertainty about the effect of thyroid hormone treatment on maternal and neonatal outcomes. ${ }^{25} \mathrm{On}$ the basis of our findings, continuing to offer thyroid hormone treatment to decrease the risk of pregnancy loss in pregnant women with TSH concentrations of $4.1-10.0 \mathrm{mIU} / \mathrm{L}$ is reasonable. Owing to the smaller magnitude of effect in the group with TSH concentrations of 2.5-4.0 mIU/L, and in light of the possible increased risk of other adverse events, treatment 
may need to be withheld in this group and guidelines may need to be revised. In fact, in the 2016 draft Guidelines for Subclinical Hypothyroidism in Pregnancy presented at the 2016 Endocrine Society meeting, levothyroxine treatment is recommended for women positive for thyroid peroxidase antibody if TSH is above $4.0 \mathrm{mIU} / \mathrm{L}$ (strong recommendation; moderate quality evidence) and may be considered if TSH is above $2.5 \mathrm{mIU} / \mathrm{L}$ (weak recommendation; low quality evidence) or for thyroid peroxidase antibody negative women (weak recommendation; low quality evidence). Moreover, results from large randomized trials such as the TSH study and the pregnancy complication data from the Controlled Antenatal Thyroid Screening study are expected soon and are likely to provide additional scientific rationale for management of subclinical hypothyroidism during pregnancy.

\section{Strengths and limitations of study}

The main limitations of this study stem from its retrospective observational design and use of administrative claims data-specifically, the potential for misclassification of treatment and confounders, lack of clinical detail, and selection biases related to health plan enrollment, diagnostic testing, and treatment choice. Methods used in the study, particularly the use of ICD-9-CM codes to ascertain adverse pregnancy outcomes, have not been previously validated. We used filled prescriptions for ascertainment of exposure, and non-adherence would bias our results toward no effect of the drug. However, because these diagnoses are assigned in the context of clinical evaluation, we expect their specificity to be high. OptumLabs Data Warehouse, like any large database of patient care, is unlikely to capture very early miscarriages, many of which are not recognized clinically.

We did not incorporate thyroxine concentrations into the study because they were not consistently included in our dataset. In routine clinical practice, thyroxine concentrations are rarely checked in pregnant women unless overt hypothyroidism is strongly suspected, ${ }^{2}$ partly owing to the limitations of available assays. Inadequate information about thyroid peroxidase antibody concentrations did not allow a stratified analysis on the basis of antibody positivity. This is an important limitation as thyroid peroxidase antibody positivity in pregnant women with subclinical hypothyroidism has been associated with worse outcomes. ${ }^{26}$ Similarly, limited information on the trajectory of TSH concentrations during treatment did not allow further analyses comparing high-normal and low-normal TSH target concentrations. Data on gestational age, body mass index, education, and smoking status, which could affect pregnancy outcomes, were also unavailable. In addition, the study population includes patients with commercial health insurance; whether our estimates are generalizable to uninsured or Medicaid populations is unclear. Finally, owing to the non-randomized nature of observational studies, we are not able to draw any causal inference. Despite careful adjustment for multiple confounders, most notably socioeconomic characteristics, residual unmeasured confounding is possible.

Nonetheless, this is the largest national study of thyroid hormone treatment among pregnant women with subclinical hypothyroidism. The large sample size enabled a stratified analysis by TSH concentration that was clinically meaningful. Despite the lack of information about the exact gestational age at time of TSH testing, the distribution of days between the TSH test and end of pregnancy suggests that the test was done early in pregnancy with subsequent early initiation of treatment. In addition, our study cohort was not restricted to academic centers and was geographically and racially diverse, thereby reflecting real world estimates in the US and implications of thyroid hormone use in pregnant women with subclinical hypothyroidism.

\section{Conclusions}

To facilitate the decision making process for pregnant women with subclinical hypothyroidism, clinicians are encouraged to use a shared decision making approach. ${ }^{27}$ With this approach, clinicians can discuss with patients the uncertainty behind our treatment recommendations and explore what is important to them when making decisions about their health with the goal of reaching a decision about treatment that best fits their situation. The timing of thyroid hormone initiation is another important area of research. Given that the observed benefit of thyroid hormone use was related to decreased risk of pregnancy loss, an event most commonly occurring in early pregnancy, treatment may be needed only in the first trimester of pregnancy. Finally, if treatment is started, monitoring of thyroid function and adjustment of treatment dose are essential.

In conclusion, this is the first national study to evaluate the effectiveness and safety of thyroid hormone for pregnant women with subclinical hypothyroidism. Thyroid hormone use was associated with a decreased risk of pregnancy loss in this population, especially those with TSH concentrations of 4.1-10 $\mathrm{mIU} / \mathrm{L}$. Further research is needed to understand whether a causal mechanism exists behind this association. In addition, the increased risk of other adverse outcomes calls for randomized trials evaluating the safety of thyroid hormone treatment in this population. These results could facilitate an informed conversation between patients and clinicians about starting thyroid hormone treatment.

Contributors: SM conceived and designed the study with input from NMSO, RGM, JPB, MNS, and VMM. RM, XY, and LRS carried out data collection and statistical analysis, with input from SM. SM wrote the manuscript, and all authors contributed to critical appraisal and review of the manuscript. All authors reviewed and agreed on the final version of the manuscript. SM and VMM are the guarantors.

Funding: This study was funded by the Mayo Clinic Robert D. and Patricia E. Kern Center for the Science of Health Care Delivery. The funders had no role in study design; in the collection, analysis, and interpretation of data; in the writing of the report; or in the decision to submit the article for publication.

Competing interests: All authors have completed the ICMJE uniform disclosure form at www.icmje.org/coi_disclosure.pdf (available on request from the corresponding author) and declare: support from the Agency for Healthcare Research and Quality and AcademyHealth for the submitted work; no financial relationships with any organizations that might have an interest in the submitted work in the previous three years; no other relationships or activities that could appear to have influenced the submitted work.

Ethical approval: Study data were statistically de-identified and accessed according to the Health Insurance Portability and Accountability Act 164.514 privacy rule. The Mayo Clinic Institutional Review Board exempted this study from approval as it represents research on pre-existing, de-identified data. Participants' consent was not obtained because all presented data were anonymized before dataset creation and risk of identification is low.

Data sharing: No additional data available.

Transparency statement: The lead authors (the manuscript's guarantors) affirm that this manuscript is an honest, accurate, and transparent account of the study being reported; that no important aspects of the study have been omitted; and that any discrepancies from the study as planned (and, if relevant, registered) have been explained. 


\section{What is already known on this topic}

Observational studies have suggested that subclinical hypothyroidism in pregnancy is associated with adverse pregnancy outcomes Insufficient evidence exists supporting net benefit of thyroid hormone treatment among pregnant women with subclinical hypothyroidism Despite the lack of evidence, current guidelines recommend levothyroxine treatment for pregnant women with subclinical hypothyroidism

\section{What this study adds}

In a national US cohort of 5405 pregnant women with subclinical hypothyroidism, only $16 \%$ of women received thyroid hormone treatment Thyroid hormone treatment was associated with decreased risk of pregnancy loss but also with increased risk of important complications such as preterm delivery, gestational diabetes, and pre-eclampsia

The benefit of thyroid hormone use on pregnancy loss was observed only among women with pre-treatment thyroid stimulating hormone concentrations of $4.1-10.0 \mathrm{mIU} / \mathrm{L}$, not 2.5-4.0 mIU/L

1 Stagnaro-Green A, Abalovich M, Alexander E, et al. American Thyroid Association Taskforce on Thyroid Disease During Pregnancy and Postpartum. Guidelines of the American Thyroid Association for the diagnosis and management of thyroid disease during pregnancy and postpartum. Thyroid 2011;21:1081-125. doi:10.1089/thy.2011.0087 pmid: 21787128.

2 Blatt AJ, Nakamoto JM, Kaufman HW. National status of testing for hypothyroidism during pregnancy and postpartum. J Clin Endocrinol Metab 2012;97:777-84. doi:10.1210/jc.20112038 pmid:22170721.

3 Aguayo A, Grau G, Vela A, et al. Urinary iodine and thyroid function in a population of healthy pregnant women in the North of Spain. J Trace Elem Med Biol 2013;27:302-6. doi:10.1016/j.jtemb.2013.07.002 pmid:23992867.

4 Negro R, Stagnaro-Green A. Diagnosis and management of subclinical hypothyroidism in pregnancy. BMJ 2014;349:g4929. doi:10.1136/bmj.g4929 pmid:25288580.

5 Chan S, Boelaert K. Optimal management of hypothyroidism, hypothyroxinaemia and euthyroid TPO antibody positivity preconception and in pregnancy. Clin Endocrinol (Oxf) 2015;82:313-26. doi:10.1111/cen.12605 pmid:25200555.

6 Sheehan PM, Nankervis A, Araujo Júnior E, Da Silva Costa F. Maternal thyroid disease and preterm birth: systematic review and meta-analysis. J Clin Endocrinol Metab 2015;100:4325-31. doi:10.1210/jc.2015-3074 pmid:26383905.

7 Maraka S, Ospina NM, O'Keeffe DT, et al. Subclinical hypothyroidism in pregnancy: a systematic review and meta-analysis. Thyroid 2016;26:580-90. doi:10.1089/thy.2015. 0418 pmid:26837268

8 De Groot L, Abalovich M, Alexander EK, et al. Management of thyroid dysfunction during pregnancy and postpartum: an Endocrine Society clinical practice guideline. J Clin Endocrinol Metab 2012;97:2543-65. doi:10.1210/jc.2011-2803 pmid:22869843.

9 Lazarus J, Brown RS, Daumerie C, Hubalewska-Dydejczyk A, Negro R, Vaidya B. 2014 European thyroid association guidelines for the management of subclinical hypothyroidism in pregnancy and in children. Eur Thyroid J 2014;3:76-94. doi:10.1159/000362597 pmid: 25114871.

10 Center for Disease Control and Prevention. Birth data. 2015. http://www.cdc.gov/nchs/ births.htm.

11 Wallace PJ, Shah ND, Dennen T, Bleicher PA, Crown WH. Optum Labs: building a novel node in the learning health care system. Health Aff (Millwood) 2014;33:1187-94. doi:10. 1377/hlthaff.2014.0038 pmid:25006145.

12 Optum. Real world health care experiences. 2015. https://www.optum.com/content/dam/ optum/resources/productSheets/5302_Data_Assets_Chart_Sheet_ISPOR.pdf.

13 Dashe JS, Casey BM, Wells CE, et al. Thyroid-stimulating hormone in singleton and twin pregnancy: importance of gestational age-specific reference ranges. Obstet Gynecol 2005:106:753-7. doi:10.1097/01.AOG.0000175836.41390.73 pmid:16199632.

14 Hershman DL, Tsui J, Wright JD, Coromilas EJ, Tsai WY, Neugut Al. Household net worth, racial disparities, and hormonal therapy adherence among women with early-stage breast cancer. J Clin Oncol 2015;33:1053-9. doi:10.1200/JCO.2014.58.3062 pmid: 25691670.

15 Deyo RA, Cherkin DC, Ciol MA. Adapting a clinical comorbidity index for use with ICD-9-CM administrative databases. J Clin Epidemiol 1992;45:613-9. doi:10.1016/0895-4356(92) 90133-8 pmid: 1607900

16 Stirrat GM. Recurrent miscarriage. Lancet 1990;336:673-5. doi:10.1016/0140-6736(90) 92159-F pmid:1975862.
17 Wang S, Teng WP, Li JX, Wang WW, Shan ZY. Effects of maternal subclinical hypothyroidism on obstetrical outcomes during early pregnancy. J Endocrinol Invest 2012;35:322-5.pmid:21642766

18 Negro R, Schwartz A, Gismondi R, Tinelli A, Mangieri T, Stagnaro-Green A. Universal screening versus case finding for detection and treatment of thyroid hormonal dysfunction during pregnancy. J Clin Endocrinol Metab 2010;95:1699-707. doi:10.1210/jc.20092009 pmid:20130074

19 Maraka S, Singh Ospina NM, O'Keeffe DT, et al. Effects of levothyroxine therapy on pregnancy outcomes in women with subclinical hypothyroidism. Thyroid 2016;26:980-6. doi:10.1089/thy.2016.0014 pmid:27112035.

20 Haymart MR. The role of clinical guidelines in patient care: thyroid hormone replacement in women of reproductive age. Thyroid 2010;20:301-7. doi:10.1089/thy.2009.0321 pmid: 20146654.

21 Singh Ospina N, Rodriguez-Gutierrez R, Brito JP, Young WF Jr, , Montori VM. Is the endocrine research pipeline broken? A systematic evaluation of the Endocrine Society clinical practice guidelines and trial registration. BMC Med 2015;13:187. doi:10.1186/ clinical practice guidelines and trial regist

22 Vaidya B, Hubalewska-Dydejczyk A, Laurberg P, Negro R, Vermiglio F, Poppe K. Treatment and screening of hypothyroidism in pregnancy: results of a European survey. Eur J Endocrinol 2012;166:49-54. doi:10.1530/EJE-11-0729 pmid:22023792.

23 Briesacher BA, Andrade SE, Fouayzi $\mathrm{H}$, Chan KA. Comparison of drug adherence rates among patients with seven different medical conditions. Pharmacotherapy 2008;28:437-43. doi:10.1592/phco.28.4.437 pmid:18363527.

24 Maruo T, Matsuo H, Mochizuki M. Thyroid hormone as a biological amplifier of differentiated trophoblast function in early pregnancy. Acta Endocrinol (Copenh) 1991;125:58-66.pmid: 1872126

25 Maraka S, O'Keeffe DT, Montori VM. Subclinical Hypothyroidism During Pregnancy-Should You Expect This When You Are Expecting?: A Teachable Moment. JAMA Intern Med 2015;175:1088-9. doi:10.1001/jamainternmed.2015.1348 pmid:25938470.

26 Liu H, Shan Z, Li C, et al. Maternal subclinical hypothyroidism, thyroid autoimmunity, and the risk of miscarriage: a prospective cohort study. Thyroid 2014;24:1642-9. doi:10.1089/ thy.2014.0029 pmid:25087688.

27 Rodriguez-Gutierrez R, Gionfriddo MR, Ospina NS, et al. Shared decision making in endocrinology: present and future directions. Lancet Diabetes Endocrinol 2016;4:706-16. doi:10.1016/S2213-8587(15)00468-4 pmid:26915314.

\section{Accepted: 12122016}

Published by the BMJ Publishing Group Limited. For permission to use (where not already granted under a licence) please go to http://group.bmj.com/group/rights-licensing/ permissions

This is an Open Access article distributed in accordance with the Creative Commons Attribution Non Commercial (CC BY-NC 3.0) license, which permits others to distribute, remix, adapt, build upon this work non-commercially, and license their derivative works on different terms, provided the original work is properly cited and the use is non-commercial. See: http://creativecommons.org/licenses/by-nc/3.0/. 


\section{Tables}

Table 1| Baseline characteristics of patients. Values are numbers (percentages) unless stated otherwise

\begin{tabular}{|c|c|c|c|c|}
\hline \multirow[b]{2}{*}{ Characteristics } & \multicolumn{2}{|c|}{ Thyroid hormone treatment } & \multirow[b]{2}{*}{ Total $(n=5405)$} & \multirow[b]{2}{*}{$P$ value } \\
\hline & Yes $(n=843)$ & No $(n=4562)$ & & \\
\hline Age: & & & & 0.08 \\
\hline Mean (SD) & $31.7(4.7)$ & $31.3(5.2)$ & $31.4(5.1)$ & \\
\hline Median (IQR) & $32(29.0-35.0)$ & $31(28.0-35.0)$ & $31(28.0-35.0)$ & \\
\hline Age group, years: & & & & 0.01 \\
\hline $18-24$ & $49(5.8)$ & $432(9.5)$ & $481(8.9)$ & \\
\hline $25-34$ & $571(67.7)$ & $2944(64.5)$ & $3515(65.0)$ & \\
\hline $35-44$ & $220(26.1)$ & $1163(25.5)$ & $1383(25.6)$ & \\
\hline $45-54$ & $3(0.4)$ & $23(0.5)$ & $26(0.5)$ & \\
\hline TSH concentration, mIU/L: & & & & $<0.01$ \\
\hline Mean (SD) & $4.8(1.7)$ & $3.3(0.9)$ & $3.5(1.2)$ & \\
\hline Median (IQR) & $4.6(3.4-5.7)$ & $3.1(2.7-3.6)$ & $3.2(2.8-3.9)$ & \\
\hline TSH concentration group, mlU/L: & & & & $<0.01$ \\
\hline $2.5-4.0$ & $330(39.1)$ & $3865(84.7)$ & 4195 (77.6) & \\
\hline 4.1-10.0 & $513(60.9)$ & $697(15.3)$ & $1210(22.4)$ & \\
\hline Ethnicity: & & & & $<0.01$ \\
\hline White & $465(55.2)$ & $2686(58.9)$ & $3151(58.3)$ & \\
\hline Black & $49(5.8)$ & $344(7.5)$ & $393(7.3)$ & \\
\hline Hispanic & $100(11.9)$ & $747(16.4)$ & $847(15.7)$ & \\
\hline Asian & $188(22.3)$ & $614(13.5)$ & $802(14.8)$ & \\
\hline Unknown & $41(4.9)$ & $171(3.7)$ & $212(3.9)$ & \\
\hline Charlson index: & & & & 0.77 \\
\hline 0 & $744(88.3)$ & $4001(87.7)$ & 4745 (87.8) & \\
\hline 1 & $79(9.4)$ & $461(10.1)$ & $540(10.0)$ & \\
\hline$\geq 2$ & $20(2.4)$ & $100(2.2)$ & $120(2.2)$ & \\
\hline Household income: & & & & 0.02 \\
\hline$<\$ 40000$ & $90(10.7)$ & $506(11.1)$ & $596(11.0)$ & \\
\hline$\$ 40000-\$ 49999$ & $41(4.9)$ & $295(6.5)$ & $336(6.2)$ & \\
\hline$\$ 50000-\$ 59999$ & $63(7.5)$ & $290(6.4)$ & $353(6.5)$ & \\
\hline$\$ 60000-\$ 74999$ & $77(9.1)$ & $503(11.0)$ & $580(10.7)$ & \\
\hline$\$ 75000-\$ 69999$ & $139(16.5)$ & $776(17.0)$ & 915 (16.9) & \\
\hline$\geq \$ 100000$ & $309(36.7)$ & $1686(37.0)$ & 1995 (36.9) & \\
\hline Unknown & $124(14.7)$ & $506(11.1)$ & $630(11.7)$ & \\
\hline Region: & & & & $<0.01$ \\
\hline Midwest & $64(7.6)$ & $416(9.1)$ & $480(8.9)$ & \\
\hline Northeast & $215(25.5)$ & $873(19.1)$ & $1088(20.1)$ & \\
\hline South & $369(43.8)$ & $2404(52.7)$ & $2773(51.3)$ & \\
\hline West & $195(23.1)$ & $869(19.0)$ & 1064 (19.7) & \\
\hline Obesity & $52(6.2)$ & $224(4.9)$ & $276(5.1)$ & 0.13 \\
\hline Diabetes mellitus & $24(2.8)$ & $127(2.8)$ & $151(2.8)$ & 0.92 \\
\hline Hypertension & $22(2.6)$ & $221(4.8)$ & $243(4.5)$ & $<0.01$ \\
\hline History of recurrent pregnancy loss & $23(2.7)$ & $49(1.1)$ & $72(1.3)$ & $<0.01$ \\
\hline History of thyroid disease & $52(6.2)$ & $153(3.4)$ & $205(3.8)$ & $<0.01$ \\
\hline
\end{tabular}

$\mathrm{IQR}=$ interquartile range; $\mathrm{TSH}=$ thyroid stimulating hormone.

${ }^{*}{ }^{2}$ test; $t$ test for continuous variables (age, TSH concentration). 


\begin{tabular}{|c|c|c|c|c|c|}
\hline \multirow[b]{2}{*}{ Adverse outcomes } & \multicolumn{2}{|c|}{ No (\%) events } & \multicolumn{2}{|c|}{ Odds ratio $(95 \% \mathrm{Cl})$} & \multirow[b]{2}{*}{ P value ${ }^{\dagger}$} \\
\hline & $\begin{array}{l}\text { Thyroid hormone } \\
\text { treatment }(n=843)\end{array}$ & $\begin{array}{l}\text { No thyroid hormone } \\
\text { treatment }(n=4562)\end{array}$ & Unadjusted & Adjusted & \\
\hline Pregnancy loss $^{\ddagger}$ & $89(10.6)$ & $614(13.5)$ & $0.76(0.60$ to 0.96$)$ & 0.62 (0.48 to 0.82$)$ & $<0.01$ \\
\hline Preterm delivery & $60(7.1)$ & $236(5.2)$ & 1.41 (1.05 to 1.88$)$ & 1.60 (1.14 to 2.24$)$ & 0.01 \\
\hline Preterm labor & $111(13.2)$ & $569(12.5)$ & $1.08(0.87$ to 1.34$)$ & $1.14(0.89$ to 1.46$)$ & 0.29 \\
\hline $\begin{array}{l}\text { Premature rupture of } \\
\text { membranes }\end{array}$ & $42(5.0)$ & $220(4.8)$ & 1.04 (0.74 to 1.45$)$ & 0.97 (0.66 to 1.42$)$ & 0.87 \\
\hline Placental abruption & $7(0.8)$ & $36(0.8)$ & 1.05 (0.47 to 2.37$)$ & $1.60(0.65$ to 3.93$)$ & 0.30 \\
\hline Gestational diabetes & $101(12.0)$ & $401(8.8)$ & $1.41(1.12$ to 1.78$)$ & 1.37 (1.05 to 1.79$)$ & 0.02 \\
\hline Gestational hypertension ${ }^{\S}$ & $49(5.8)$ & $221(4.8)$ & 1.24 (0.90 to 1.70$)$ & 1.27 (0.88 to 1.82$)$ & 0.21 \\
\hline Pre-eclampsia $^{\S}$ & $46(5.5)$ & $177(3.9)$ & $1.43(1.03$ to 2.00$)$ & 1.61 (1.10 to 2.37$)$ & 0.01 \\
\hline Poor fetal growth & $78(9.3)$ & $397(8.7)$ & 1.07 (0.83 to 1.38$)$ & $1.12(0.84$ to 1.50$)$ & 0.45 \\
\hline Tachycardia & $18(2.1)$ & $90(2.0)$ & $1.08(0.65$ to 1.81$)$ & $1.77(1.00$ to 3.11$)$ & 0.05 \\
\hline \multicolumn{6}{|c|}{$\begin{array}{l}\text { *Adjusted for age and thyroid stimulating hormone (TSH) concentration as continuous variables and for ethnicity, income, Charlson index, hypertension, obesi } \\
\text { and history of thyroid disease. } \\
\dagger \text { For adjusted outcomes. }\end{array}$} \\
\hline
\end{tabular}


Table 3| Interaction of pre-treatment thyroid stimulating hormone concentration (continuous variable) with thyroid hormone treatment effect on clinical outcomes

\begin{tabular}{|c|c|}
\hline Adverse outcomes & $P$ value for interaction \\
\hline Pregnancy loss ${ }^{\dagger}$ & 0.01 \\
\hline Preterm delivery & 0.62 \\
\hline Preterm labor & 0.97 \\
\hline Premature rupture of membranes & 0.51 \\
\hline Placental abruption & 0.84 \\
\hline Gestational diabetes & 0.66 \\
\hline Gestational hypertension ${ }^{\ddagger}$ & 0.07 \\
\hline Pre-eclampsia ${ }^{\ddagger}$ & 0.22 \\
\hline Poor fetal growth & 0.69 \\
\hline Tachycardia & 0.41 \\
\hline
\end{tabular}

*Adjusted for age, ethnicity, income, Charlson index, hypertension, obesity, and history of thyroid disease. †Additionally adjusted for history of pregnancy loss.

$\ddagger$ Adjusted for age, ethnicity, income, Charlson index, obesity, and history of thyroid disease. 
Table 4| Clinical outcomes associated with thyroid hormone treatment, stratified by thyroid stimulating hormone (TSH) concentration

\begin{tabular}{|c|c|c|c|}
\hline \multirow[b]{2}{*}{ Adverse outcomes } & \multicolumn{2}{|c|}{ Odds ratio $(95 \% \mathrm{Cl})^{\dagger}$} & \multirow[b]{2}{*}{$P$ value for Interaction } \\
\hline & TSH 2.5-4.0 mlU/L & TSH 4.1-10.0 mIU/L & \\
\hline Pregnancy loss ${ }^{\ddagger}$ & 0.91 (0.65 to 1.23$)$ & 0.45 (0.30 to 0.65$)$ & $<0.01$ \\
\hline Preterm delivery & 1.90 (1.26 to 2.86$)$ & $1.37(0.82$ to 2.29$)$ & 0.33 \\
\hline Preterm labor & 1.25 (0.91 to 1.72$)$ & 1.01 (0.71 to 1.43$)$ & 0.37 \\
\hline Premature rupture of membranes & 1.03 (0.61 to 1.75$)$ & 0.88 (0.53 to 1.46$)$ & 0.67 \\
\hline Placental abruption & $1.42(0.49$ to 4.07$)$ & $2.16(0.36$ to 13.03$)$ & 0.69 \\
\hline Gestational diabetes & 1.40 (0.99 to 1.99$)$ & $1.48(1.00$ to 2.18$)$ & 0.85 \\
\hline Gestational hypertension $^{\S}$ & $1.76(1.13$ to 2.74$)$ & 0.86 (0.51 to 1.45$)$ & 0.04 \\
\hline Pre-eclampsia ${ }^{\S}$ & 1.75 (1.08 to 2.84$)$ & 1.61 (0.91 to 2.88$)$ & 0.83 \\
\hline Poor fetal growth & 0.96 (0.63 to 1.45$)$ & $1.10(0.74$ to 1.63$)$ & 0.65 \\
\hline Tachycardia & 1.61 (0.84 to 3.09$)$ & 1.46 (0.51 to 4.21$)$ & 0.88 \\
\hline
\end{tabular}

*Adjusted for age, ethnicity, income, Charlson index, hypertension, obesity, and history of thyroid disease. †Reference group is untreated women.

$\ddagger$ Additionally adjusted for history of pregnancy loss.

$\S$ Adjusted for age, ethnicity, income, Charlson index, obesity, and history of thyroid disease. 


\section{Figures}

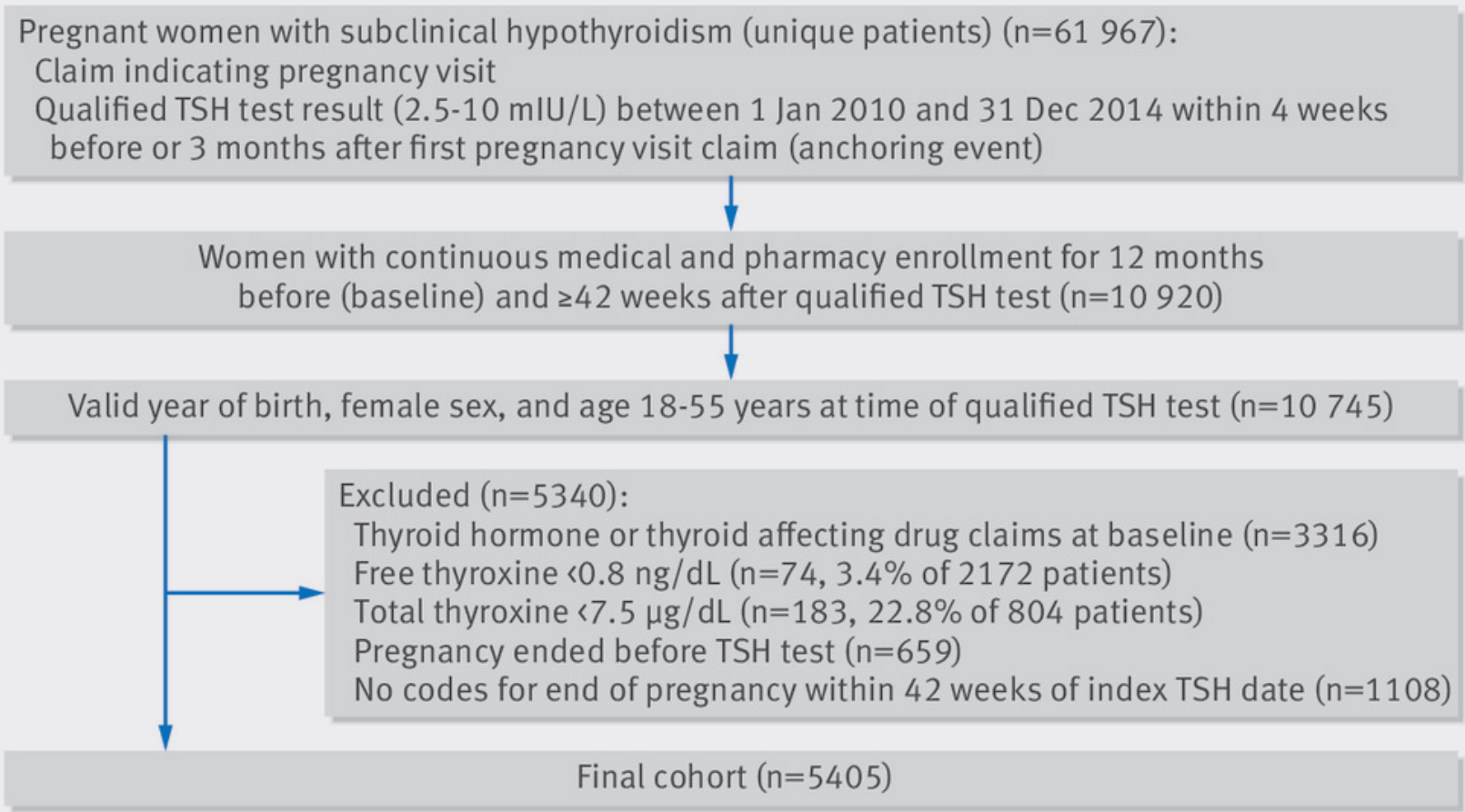

Fig 1 Cohort creation flowchart 

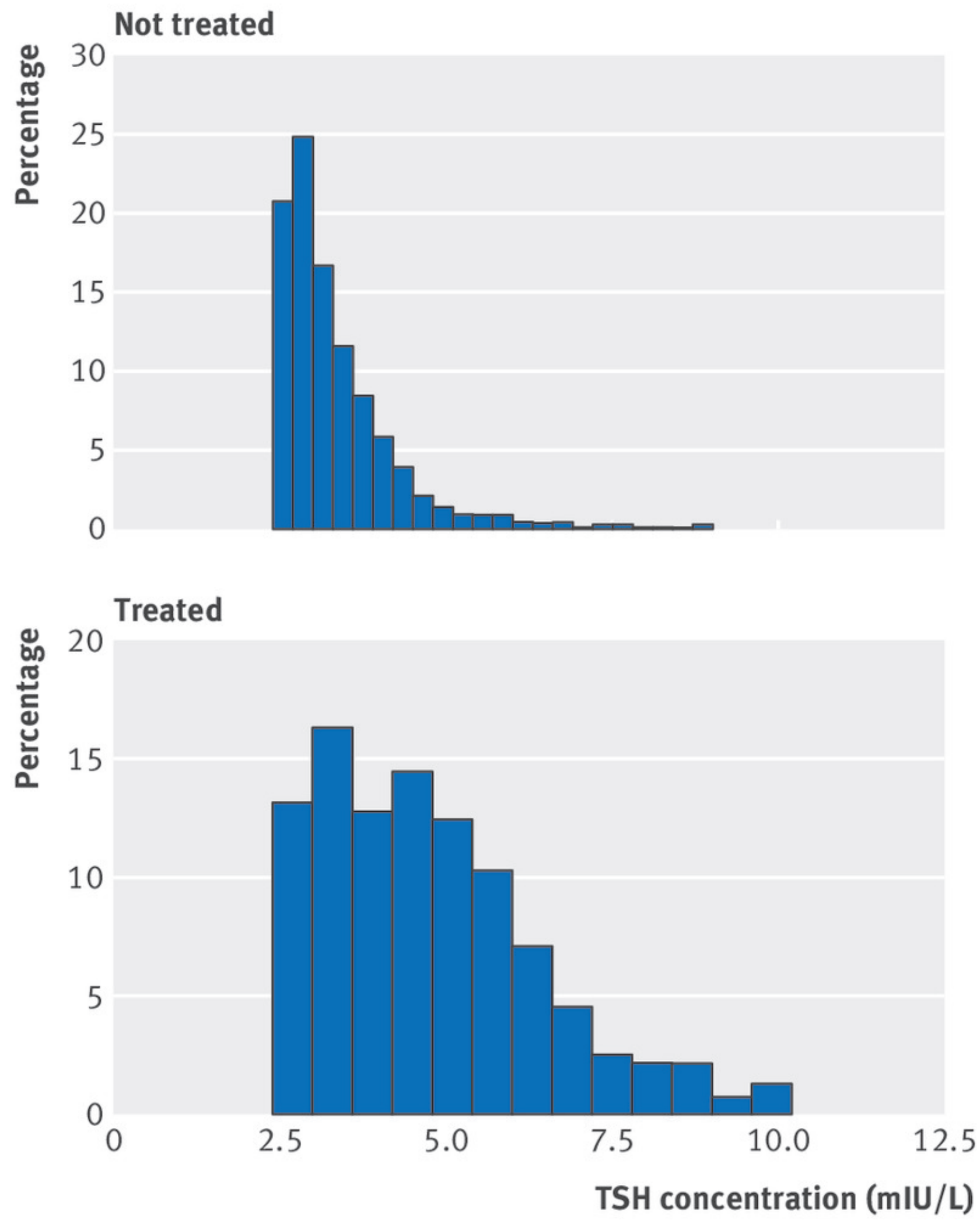

Fig 2 Distribution of thyroid stimulating hormone (TSH) in treated and untreated groups 